\title{
To improve the "target" of transanal hemorrhoidal dearterialization
}

\author{
C. Ratto $\cdot$ A. Parello
}

Received: 26 December 2010/ Accepted: 16 March 2011/Published online: 20 April 2011

(c) Springer-Verlag 2011

Sir,

We read with interest the paper by Toh et al. [1]. This study was carried out with the critical attitude of those who want to improve a surgical treatment that is very effective but can still be improved. Our comments about the study results concern the "functional anatomy" of the lower rectum. Actually, in addition to the exact number of terminal branches of the higher rectal arteries, a fundamental factor in this surgical approach is the correct understanding of the topography of these branches and their relationship with the rectal wall. Hemorrhoidal dearterialization with transanal hemorrhoidal dearterialization (THD) ensures an excellent control of rectal bleeding, and in most cases, the resolution of prolapse thanks to a "tailored mucopexy". The complex vascular network of the "corpus cavernosum recti" is the key to perfecting this therapeutic approach. According to our experience, there are always more than three (usually six) terminal branches of hemorrhoidal arteries to be ligated [2]. The target of therapy should be the point at which the arteries enter the submucosa of the low rectum, just before supplying blood to the "corpus cavernosum recti" [3]. In this way, an extraparietal arterial ligation can be avoided, and action can be taken right where the arterial hyperflow could have the greatest effect on the hemorrhoids. Use of Doppler-guided THD allows a detailed identification of the arteries and a rigorous and effective ligation of them. Moreover, the surgeon avoids taking big bites when suturing and making a "blind dearterialization" that would be at greater risk of bleeding and might partially alter both the anatomy and dynamics of the rectal wall, thus affecting rectal compliance and causing postoperative functional disturbances.

\section{References}

1. Toh EL, Ng KH, Eu KW (2010) The fourth branch of the superior rectal artery and its significance in transanal haemorrhoidal dearterialisation. Tech Coloproctol 14:345-348

2. Ratto C, Donisi L, Parello A, Litta F, Doglietto GB (2010) Evaluation of transanal hemorrhoidal dearterialization as a minimally invasive therapeutic approach to hemorrhoids. Dis Colon Rectum 53:803-811

3. Ratto C, Parello A, Zaccone G, Donisi L, Litta F, Doglietto GB (2010) Assessment of hemorrhoidal arteries location within the rectum using endorectal ultrasound and echo-color Doppler. Dis Colon Rectum 53:578
C. Ratto $(\bowtie) \cdot$ A. Parello

Catholic University, Rome, Italy

e-mail: carloratto@tiscali.it 